\title{
Fractional maximal operator and its higher order commutators in generalized weighted Morrey spaces on Heisenberg group
}

\author{
Javanshir V. Azizov *
}

Received: 21.06.2019 / Revised: 29.11.2019 / Accepted: 08.02.20

\begin{abstract}
In this paper we study Spanne-Guliyev type boundedness of the fractional maximal operator $M_{\alpha}, 0 \leq \alpha<Q$ on Heisenberg group $\mathbb{H}_{n}$ in the generalized weighted Morrey spaces $M_{p, \varphi}\left(\mathbb{H}_{n}, w\right)$ including weak versions, where $Q$ is the homogeneous dimension of $\mathbb{H}_{n}$. In the case $b \in B M O\left(\mathbb{H}_{n}\right)$ we obtain Spanne-Guliyev type boundedness of the kth-order fractional maximal commutator operator $M_{b, \alpha, k}$ on the generalized weighted Morrey spaces.
\end{abstract}

Keywords. Heisenberg group, fractional maximal operator, generalized weighted Morrey space, commutator, $B M O$.

Mathematics Subject Classification (2010): Primary 42B25, 42B35, 43A15, 43A80

\section{Introduction}

The classical Morrey spaces were introduced by Morrey [27] to study the local behavior of solutions to second-order elliptic partial differential equations. Moreover, various Morrey spaces are defined in the process of study. Guliyev, Mizuhara and Nakai [11,26, 28] introduced generalized Morrey spaces $M_{p, \varphi}\left(\mathbb{R}^{n}\right)$ (see, also [12,13,30]). Komori and Shirai [23] defined weighted Morrey spaces $L_{p, \kappa}(w)$. Guliyev [15] gave a concept of the generalized weighted Morrey spaces $M_{p, \varphi}\left(\mathbb{R}^{n}, w\right)$ which could be viewed as extension of both $M_{p, \varphi}\left(\mathbb{R}^{n}\right)$ and $L_{p, \kappa}(w)$. In [15], the boundedness of the classical operators and their commutators in spaces $M_{p, \varphi}\left(\mathbb{R}^{n}, w\right)$ was also studied, see also [16-22].

The spaces $M_{p, \varphi}\left(\mathbb{R}^{n}, w\right)$ defined by the norm

$$
\|f\|_{M_{p, \varphi}\left(\mathbb{R}^{n}, w\right)} \equiv \sup _{x \in \mathbb{R}^{n}, r>0} \varphi(x, r)^{-1} w(B(x, r))^{-1 / p}\|f\|_{L_{p}(B(x, r), w)},
$$

where the function $\varphi$ is a positive measurable function on $\mathbb{R}^{n} \times(0, \infty)$ and $w$ is a nonnegative measurable function on $\mathbb{R}^{n}$.

Heisenberg groups, in discrete and continuous versions, appear in many parts of mathematics, including Fourier analysis, several complex variables, geometry, and topology. We state some basic results about Heisenberg group. More detailed information can be found

\footnotetext{
* Corresponding author
} 
in [5-7] and the references therein. Let $\mathbb{H}_{n}$ be the $2 n+1$-dimensional Heisenberg group. That is, $\mathbb{H}_{n}=\mathbb{C}^{n} \times \mathbb{R}$, with multiplication

$$
(z, t) \cdot(w, s)=(z+w, t+s+2 \operatorname{Im}(z \cdot \bar{w}))
$$

where $z \cdot \bar{w}=\sum_{j=1}^{n} z_{j} \bar{w}_{j}$. The inverse element of $u=(z, t)$ is $u^{-1}=(-z,-t)$ and we write the identity of $\mathbb{H}_{n}$ as $0=(0,0)$. The Heisenberg group is a connected, simply connected nilpotent Lie group. We define one-parameter dilations on $\mathbb{H}_{n}$, for $r>0$, by $\delta_{r}(z, t)=$ $\left(r z, r^{2} t\right)$. These dilations are group automorphisms and the Jacobian determinant is $r^{Q}$, where $Q=2 n+2$ is the homogeneous dimension of $\mathbb{H}_{n}$. A homogeneous norm on $\mathbb{H}_{n}$ is given by

$$
|(z, t)|=\left(|z|^{2}+|t|\right)^{1 / 2} .
$$

With this norm, we define the Heisenberg ball centered at $u=(z, t)$ with radius $r$ by $B(u, r)=\left\{v \in \mathbb{H}_{n}:\left|u^{-1} v\right|<r\right\}$, and we denote by $B(u, 2 r)=\left\{y \in \mathbb{H}_{n}:\left|u^{-1} 2 v\right|<r\right\}$ the open ball centered at $u$, with radius $2 r$. The volume of the ball $B(u, r)$ is $C_{Q} r^{Q}$, where $C_{Q}$ is the volume of the unit ball $B(0,1)$.

Using coordinates $u=(z, t)=(x+i y, t)$ for points in $\mathbb{H}_{n}$, the left-invariant vector fields $X_{j}, Y_{j}$ and $T$ on $\mathbb{H}_{n}$ equal to $\frac{\partial}{\partial x_{j}}, \frac{\partial}{\partial y_{j}}$ and $\frac{\partial}{\partial t}$ at the origin are given by

$$
X_{j}=\frac{\partial}{\partial x_{j}}+2 y_{j} \frac{\partial}{\partial t}, \quad Y_{j}=\frac{\partial}{\partial y_{j}}-2 x_{j} \frac{\partial}{\partial t}, \quad T=\frac{\partial}{\partial t},
$$

respectively. These $2 n+1$ vector fields form a basis for the Lie algebra of $\mathbb{H}_{n}$ with commutation relations

$$
\left[Y_{j}, X_{j}\right]=4 T
$$

for $j=1, \ldots, n$, and all other commutators equal to 0 .

Let $f \in L_{1}^{\text {loc }}\left(\mathbb{H}_{n}\right)$. The fractional maximal operator $M_{\alpha}$ is defined by

$$
M_{\alpha} f(u)=\sup _{r>0}|B(u, r)|^{-1+\frac{\alpha}{Q}} \int_{B(u, r)}|f(v)| d V(v), \quad 0<\alpha<Q,
$$

where $Q$ is the homogeneous dimension of the Heisenberg group $\mathbb{H}_{n}$ and $|B(u, r)|$ is the Haar measure of the $\mathbb{H}_{n}$ - ball $B(u, r)$.

If $\alpha=0$, then $M \equiv M_{\alpha}$ is the Hardy-Littlewood maximal operator on $\mathbb{H}_{n}$. The operators $M_{\alpha}$ plays an important role in real and harmonic analysis and applications (see, for example [5] and [6]).

In the present work, we study Spanne-Guliyev type boundedness of the fractional maximal operator $M_{\alpha}, 0 \leq \alpha<Q$ on Heisenberg group $\mathbb{H}_{n}$ in the generalized weighted Morrey spaces $M_{p, \varphi}\left(\mathbb{H}_{n}, w\right)$ including weak versions, where $Q$ is the homogeneous dimension of $\mathbb{H}_{n}$. In the case $b \in B M O\left(\mathbb{H}_{n}\right)$ we obtain Spanne-Guliyev type boundedness of the $k$ thorder fractional maximal commutator operator $M_{b, \alpha, k}$ on the generalized weighted Morrey spaces.

By $A \lesssim B$ we mean that $A \leq C B$ with some positive constant $C$ independent of appropriate quantities. If $A \lesssim B$ and $B \lesssim A$, we write $A \approx B$ and say that $A$ and $B$ are equivalent. 


\section{Preliminaries and some lemmas}

By a weight function, briefly weight, we mean a locally integrable function on $\mathbb{H}_{n}$ which takes values in $(0, \infty)$ almost everywhere. For a weight $w$ and a measurable set $E$, we define $w(E)=\int_{E} w(x) d x$, and denote the Lebesgue measure of $E$ by $|E|$ and the characteristic function of $E$ by $\chi_{E}$.

If $w$ is a weight function, we denote by $L_{p, w}\left(\mathbb{H}_{n}\right)$ the weighted Lebesgue space defined by finiteness of the norm

$$
\|f\|_{L_{p, w}\left(\mathbb{H}_{n}\right)}=\left(\int_{\mathbb{H}_{n}}|f(u)|^{p} w(u) d V(u)\right)^{\frac{1}{p}}<\infty, \quad \text { if } \quad 1 \leq p<\infty
$$

and

$$
\|f\|_{L_{\infty, w}\left(\mathbb{H}_{n}\right)}=\underset{u \in \mathbb{H}_{n}}{\operatorname{ess} \sup }|f(u)| w(u), \quad \text { if } \quad p=\infty .
$$

We define the generalized weighed Morrey spaces as follows.

Definition 2.1 Let $1 \leq p<\infty, \varphi$ be a positive measurable function on $\mathbb{H}_{n} \times(0, \infty)$ and $w$ be non-negative measurable function on $\mathbb{H}_{n}$. We denote by $M_{p, \varphi}\left(\mathbb{H}_{n}, w\right) \equiv M_{p, \varphi}(w)$ the generalized weighted Morrey space, the space of all functions $f \in L_{p, w}^{\text {loc }}\left(\mathbb{H}_{n}\right)$ with finite norm

$$
\|f\|_{M_{p, \varphi}(w)}=\sup _{u \in \mathbb{H}_{n}, r>0} \varphi(u, r)^{-1} w(B(u, r))^{-\frac{1}{p}}\|f\|_{L_{p, w}(B(u, r))},
$$

where $L_{p, w}(B(u, r))$ denotes the weighted $L_{p}$-space of measurable functions $f$ for which

$$
\|f\|_{L_{p, w}(B(u, r))} \equiv\left\|f \chi_{B(u, r)}\right\|_{L_{p, w}\left(\mathbb{H}_{n}\right)}=\left(\int_{B(u, r)}|f(v)|^{p} w(v) d V(v)\right)^{\frac{1}{p}} .
$$

Furthermore, by $W M_{p, \varphi}(w)$ we denote the weak generalized weighted Morrey space of all functions $f \in W L_{p, w}^{\text {loc }}\left(\mathbb{H}_{n}\right)$ for which

$$
\|f\|_{W M_{p, \varphi}(w)}=\sup _{u \in \mathbb{H}_{n}, r>0} \varphi(u, r)^{-1} w(B(u, r))^{-\frac{1}{p}}\|f\|_{W L_{p, w}(B(u, r))}<\infty,
$$

where $W L_{p, w}(B(u, r))$ denotes the weak $L_{p, w}$-space of measurable functions $f$ for which

$$
\|f\|_{W L_{p, w}(B(u, r))} \equiv\left\|f \chi_{B(u, r)}\right\|_{W L_{p, w}\left(\mathbb{H}_{n}\right)}=\sup _{t>0} t\left(\int_{\{v \in B(u, r):|f(v)|>t\}} w(v) d V(v)\right)^{\frac{1}{p}} .
$$
if

We recall a weight function $w$ is in the Muckenhoupt's class $A_{p}\left(\mathbb{H}_{n}\right)[24], 1<p<\infty$,

$$
[w]_{A_{p}}:=\sup _{B}[w]_{A_{p}(B)}=\sup _{B}\left(\frac{1}{|B|} \int_{B} w(u) d V(u)\right)\left(\frac{1}{|B|} \int_{B} w(u)^{1-p^{\prime}} d V(u)\right)^{p-1}<\infty,
$$

where the supremum is taken with respect to all the balls $B$ and $\frac{1}{p}+\frac{1}{p^{\prime}}=1$. Note that, for all balls $B$ Hölder's inequality is

$$
[w]_{A_{p}(B)}^{\frac{1}{p}}=|B|^{-1}\|w\|_{L_{1}(B)}^{\frac{1}{p}}\left\|w^{-\frac{1}{p}}\right\|_{L_{p^{\prime}}(B)} \geq 1 .
$$


For $p=1, w \in A_{1}\left(\mathbb{H}_{n}\right)$ is defined by the condition $M w(u) \leq C w(u)$ with $[w]_{A_{1}}=$ $\sup _{u \in \mathbb{H}_{n}} \frac{M w(u)}{w(u)}$, and for $p=\infty A_{\infty}\left(\mathbb{H}_{n}\right)=\cup_{1 \leq p<\infty} A_{p}\left(\mathbb{H}_{n}\right)$ and $[w]_{\infty}=\inf _{1 \leq p<\infty}[w]_{A_{p}}$.

A weight function $w$ is in the Muckenhoupt-Wheeden class $A_{p, q}\left(\mathbb{H}_{n}\right), 1<p<\infty$, if

$$
\begin{aligned}
{[w]_{A_{p, q}} } & :=\sup _{B}[w]_{A_{p, q}(B)} \\
& =\sup _{B}\left(\frac{1}{|B|} \int_{B} w(u)^{q} d V(u)\right)^{1 / q}\left(\frac{1}{|B|} \int_{B} w(u)^{-p^{\prime}} d V(u)\right)^{1 / p^{\prime}}<\infty,
\end{aligned}
$$

where the supremum is taken with respect to all the balls $B$ and $\frac{1}{p}+\frac{1}{p^{\prime}}=1$. Note that, for all balls $B$ Hölder's inequality is

$$
[w]_{A_{p, q}(B)}=|B|^{\frac{1}{p}-\frac{1}{q}-1}\|w\|_{L_{q}(B)}\left\|w^{-1}\right\|_{L_{p^{\prime}}(B)} \geq 1 .
$$

While $p=1, w \in A_{1, q}$ with $1<q<\infty$ if

$$
\begin{aligned}
{[w]_{A_{1, q}} } & :=\sup _{B}[w]_{A_{1, q}(B)} \\
& =\sup _{B}\left(\frac{1}{|B|} \int_{B} w(u)^{q} d V(u)\right)^{\frac{1}{q}}\left(\operatorname{ess} \sup _{u \in B} \frac{1}{w(u)}\right)<\infty .
\end{aligned}
$$

We denote by $B(u, 2 r)=\left\{y \in \mathbb{H}_{n}:\left|u^{-1} v\right|<2 r\right\}$.

In the sequel $\mathfrak{M}\left(\mathbb{R}_{+}\right), \mathfrak{M}^{+}\left(\mathbb{R}_{+}\right)$and $\mathfrak{M}^{+}\left(\mathbb{R}_{+} ; \uparrow\right)$ stand for the set of Lebesgue-measurable functions on $\mathbb{R}_{+}$, and its subspaces of nonnegative and nonnegative non-decreasing functions, respectively. We also denote

$$
\mathbb{A}=\left\{\varphi \in \mathfrak{M}^{+}\left(\mathbb{R}_{+} ; \uparrow\right): \lim _{t \rightarrow 0^{+}} \varphi=0\right\}
$$

Let $u$ be a continuous and non-negative function on $\mathbb{R}_{+}$. We define the supremal operator $\bar{S}_{u}$ by

$$
\left(\bar{S}_{u} g\right)(t):=\|u g\|_{L_{\infty}(t, \infty)}, \quad t \in(0, \infty) .
$$

The following theorem was proved in [4].

Theorem 2.1 [4] Suppose that $v_{1}$ and $v_{2}$ are nonnegative measurable functions such that $0<\left\|v_{1}\right\|_{L_{\infty}(0, \cdot)}<\infty$ for every $t>0$. Let $u$ be a continuous nonnegative function on $\mathbb{R}$. Then the operator $\bar{S}_{u}$ is bounded from $L_{\infty, v_{1}}\left(\mathbb{R}_{+}\right)$to $L_{\infty, v_{2}}\left(\mathbb{R}_{+}\right)$on the cone $\mathbb{A}$ if and only if

$$
\left\|v_{2} \bar{S}_{u}\left(\left\|v_{1}\right\|_{L_{\infty}(\cdot, \infty)}^{-1}\right)\right\|_{L_{\infty}\left(\mathbb{R}_{+}\right)}<\infty
$$




\section{Fractional maximal operator in the spaces $M_{p, \varphi}\left(\mathbb{H}_{n}, w\right)$}

In this section, we shall give the Spanne-Guliyev type boundedness of the operator $M_{\alpha}$ on the generalized weighted Morrey spaces $M_{p, \varphi}\left(\mathbb{H}_{n}, w\right)$, including weak versions. In the case of $\mathbb{R}^{n}$ Spanne-Guliyev type result for the operator $M_{\alpha}$ in the space $M_{p, \varphi}\left(\mathbb{R}^{n}, w\right)$ was proved in [22], see also [1,18,21].

The following Guliyev weighted local estimates are valid (see [15]).

Theorem 3.1 Let $1 \leq p<q<\infty, 0 \leq \alpha<\frac{Q}{p}, \frac{1}{q}=\frac{1}{p}-\frac{\alpha}{Q}$, and $\omega \in A_{p, q}\left(\mathbb{H}_{n}\right)$. Then, for $p>1$ the inequality

$$
\left\|M_{\alpha} f\right\|_{L_{q, w}(B(u, r))} \lesssim\left(w^{q}(B(u, r))\right)^{\frac{1}{q}} \sup _{t \geq r}\|f\|_{L_{p, w^{p}}(B(u, t)}\left(w^{q}(B(u, t))\right)^{-\frac{1}{q}}
$$

holds for any ball $B(u, r)$ and for all $f \in L_{p, w}^{l o c}\left(\mathbb{H}_{n}\right)$.

Moreover, for $p=1$ the inequality

$$
\left\|M_{\alpha} f\right\|_{W L_{q, w^{q}}(B(u, r))} \lesssim\left(w^{q}(B(u, r))\right)^{\frac{1}{q}} \sup _{t \geq r}\|f\|_{L_{1, w}(B(u, t)}\left(w^{q}(B(u, t))\right)^{-\frac{1}{q}}
$$

holds for any ball $B(u, r)$ and for all $f \in L_{1, w}^{l o c}\left(\mathbb{H}_{n}\right)$.

Proof. Let $1<p<q<\infty, 0<\alpha<\frac{Q}{p}, \frac{1}{q}=\frac{1}{p}-\frac{\alpha}{Q}$, and $w \in A_{p, q}\left(\mathbb{H}_{n}\right)$. For arbitrary $u \in \mathbb{H}_{n}$ and $r>0$, set $B=B(u, r), 2 B=B(u, 2 r)$.

We present $f$ as

$$
f=f_{1}+f_{2}, \quad f_{1}(v)=f(v) \chi_{2 B}(v), \quad f_{2}(v)=f(v) \chi_{\mathrm{c}_{(2 B)}}(v)
$$

and have

$$
\left\|M_{\alpha} f\right\|_{L_{q, w^{q}}(B)} \leq\left\|M_{\alpha} f_{1}\right\|_{L_{q, w^{q}(B)}}+\left\|M_{\alpha} f_{2}\right\|_{L_{q, w^{q}}(B)} .
$$

Since $f_{1} \in L_{p, w^{p}\left(\mathbb{H}_{n}\right)}, M_{\alpha} f_{1} \in L_{q, w^{q}}\left(\mathbb{H}_{n}\right)$ and from the boundedness of $M_{\alpha}$ from $L_{p, w^{p}}\left(\mathbb{H}_{n}\right)$ to $L_{q, w^{q}}\left(\mathbb{H}_{n}\right)$ (see [3,29]) it follows that

$$
\left\|M_{\alpha} f_{1}\right\|_{L_{q, w^{q}}(B)} \leq\left\|M_{\alpha} f_{1}\right\|_{L_{q, w^{q}}} \lesssim\left\|f_{1}\right\|_{L_{p, w^{p}}}=\|f\|_{L_{p, w^{p}(2 B)}}
$$

From (3.3) we obtain

$$
\left\|M_{\alpha} f_{1}\right\|_{L_{q, w^{q}}(B)} \lesssim\left(w^{q}(B(u, r))\right)^{\frac{1}{q}} \sup _{t \geq r}\|f\|_{L_{p, w}(B(u, t))}\left(w^{q}(B(u, t))\right)^{-\frac{1}{q}} .
$$

Let $\zeta=(h, \tau)$ be an arbitrary point in $B \equiv B(u, r)$. If $B(\zeta, t) \cap{ }^{\complement} B(u, 2 r) \neq \varnothing$, then $t>r$. Indeed, if $v \in B(\zeta, t) \cap{ }^{C} B(u, 2 r)$, then we get $t>\left|\zeta^{-1} v\right| \geq\left|v^{-1} u\right|-\left|\zeta^{-1} u\right|>$ $2 r-r=r$.

On the other hand, $B(\zeta, t) \cap{ }^{\mathrm{c}} B(u, 2 r) \subset B(u, 2 t)$. Indeed, if $v \in B(\zeta, t) \cap{ }^{\mathrm{C}} B(u, 2 r)$, then we get $\left|v^{-1} u\right| \leq\left|\zeta^{-1} v\right|+\left|\zeta^{-1} u\right|<t+r<2 t$. Hence, for all $\zeta \in B$

$$
\begin{aligned}
M_{\alpha} f_{2}(\zeta) & =\sup _{t>0}|B(\zeta, t)|^{-1+\frac{\alpha}{Q}} \int_{B(\zeta, t)}\left|f_{2}(v)\right| d V(v) \\
& \lesssim \sup _{t>r}|B(u, 2 t)|^{-1+\frac{\alpha}{Q}} \int_{B(\zeta, t) \cap{ }^{\complement} B(u, 2 r)}|f(v)| d V(v) \\
& \leq \sup _{t>r}|B(u, 2 t)|^{-1+\frac{\alpha}{Q}} \int_{B(u, 2 t)}|f(v)| d V(v) \\
& =\sup _{t>2 r}|B(u, t)|^{-1+\frac{\alpha}{Q}} \int_{B(u, t)}|f(v)| d V(v) .
\end{aligned}
$$


By applying Hölder's inequality, for all $\zeta \in B$ we get

$$
\begin{aligned}
M_{\alpha} f_{2}(\zeta) & \lesssim \sup _{t>2 r}|B(u, t)|^{-1+\frac{\alpha}{Q}} \int_{B(u, t)}|f(v)| d V(v) \\
& \lesssim \sup _{t>r}|B(u, t)|^{-1+\frac{\alpha}{Q}}\|f\|_{L_{p, w^{p}}(B(u, t))}\left\|w^{-1}\right\|_{L_{p^{\prime}}(B(u, t))} \\
& \lesssim \sup _{t>r}\|f\|_{L_{p, w^{p}}(B(u, t))}\left(w^{q}(B(u, t))\right)^{-\frac{1}{q}}
\end{aligned}
$$

Thus, the function $M_{\alpha} f_{2}(\zeta)$, with fixed $u$ and $r$, is dominated by the expression not depending on $\zeta$. Then

$$
\left\|M_{\alpha} f_{2}\right\|_{L_{q, w^{q}}(B(u, r))} \lesssim\left(w^{q}(B(u, r))\right)^{\frac{1}{q}} \sup _{t>r}\|f\|_{L_{p, w}(B(u, t))}\left(w^{q}(B(u, t))\right)^{-\frac{1}{q}} .
$$

We then obtain (3.1) from (3.4) and (3.5).

Let $p=1$, and $w \in A_{1, q}\left(\mathbb{H}_{n}\right)$.

Then,

$$
\left\|M_{\alpha} f\right\|_{W L_{q, w^{q}(B)}} \leq\left\|M_{\alpha} f_{1}\right\|_{W L_{q, w^{q}(B)}}+\left\|M_{\alpha} f_{2}\right\|_{W L_{q, w^{q}}(B)} .
$$

Since $f_{1} \in L_{1, w\left(\mathbb{H}_{n}\right)}, M_{\alpha} f_{1} \in W L_{q, w^{q}}\left(\mathbb{H}_{n}\right)$ and from the boundedness of $M_{\alpha}$ from $L_{, w}\left(\mathbb{H}_{n}\right)$ to $W L_{q, w^{q}}\left(\mathbb{H}_{n}\right)$ (see [3,29]) it follows that

$$
\left\|M_{\alpha} f_{1}\right\|_{W L_{q, w^{q}}(B)} \leq\left\|M_{\alpha} f_{1}\right\|_{W L_{q, w^{q}}} \lesssim\left\|f_{1}\right\|_{L_{1, w}}=\|f\|_{L_{1, w}(2 B)} .
$$

From (3.6) we obtain

$$
\left\|M_{\alpha} f_{1}\right\|_{W L_{q, w^{q}}(B)} \lesssim\left(w^{q}(B(u, r))\right)^{\frac{1}{q}} \sup _{t>r}\|f\|_{L_{1, w}(B(u, t))}\left(w^{q}(B(u, t))\right)^{-\frac{1}{q}} .
$$

On the other hand,

$$
\begin{aligned}
\left\|M_{\alpha} f_{2}\right\|_{W L_{q, w^{q}}(B(u, r))} & \leq\left\|M_{\alpha} f_{2}\right\|_{L_{q, w}(B(u, r))} \\
& \lesssim\left(w^{q}(B(u, r))\right)^{\frac{1}{q}} \sup _{t>r}\|f\|_{L_{1, w}(B(u, t))}\left(w^{q}(B(u, t))\right)^{-\frac{1}{q}} .
\end{aligned}
$$

We then obtain (3.2) from (3.7) and (3.8).

For the operator $M_{\alpha}$ the following Spanne-Guliyev type result on the space $M_{p, \varphi}(w)$ is valid.

Theorem 3.2 Let $1 \leq p<q<\infty, 0<\alpha<\frac{Q}{p}, \frac{1}{q}=\frac{1}{p}-\frac{\alpha}{Q}, w \in A_{p, q}\left(\mathbb{H}_{n}\right)$, and $\left(\varphi_{1}, \varphi_{2}\right)$ satisfy the condition

$$
\sup _{t>r} \frac{\underset{t<s<\infty}{\operatorname{ess} \inf } \varphi_{1}(u, s)\left(w^{p} B((u, s))\right)^{1 / p}}{\left(w^{q}(B(u, t))\right)^{1 / q}} \leq C \varphi_{2}(u, r),
$$

where $C$ does not depend on $u$ and $r$. Then the operator $M_{\alpha}$ is bounded from $M_{p, \varphi_{1}}\left(w^{p}\right)$ to $M_{q, \varphi_{2}}\left(w^{q}\right)$ for $p>1$ and from $M_{1, \varphi_{1}}(w)$ to $W M_{q, \varphi_{2}}\left(w^{q}\right)$ for $p=1$. Moreover, for $p>1$

$$
\left\|M_{\alpha} f\right\|_{M_{q, \varphi_{2}}\left(w^{q}\right)} \lesssim\|f\|_{M_{p, \varphi_{1}}\left(w^{p}\right)}
$$

and for $p=1$

$$
\left\|M_{\alpha} f\right\|_{W M_{q, \varphi_{2}}\left(w^{q}\right)} \lesssim\|f\|_{M_{1, \varphi_{1}}(w)} .
$$


Proof. For $p>1$ from Theorem 2.1 and Theorem 3.1 we get

$$
\begin{aligned}
\left\|M_{\alpha} f\right\|_{M_{q, \varphi_{2}}\left(w^{q}\right)} & \lesssim \sup _{u \in \mathbb{H}_{n}, r>0} \varphi_{2}(u, r)^{-1} \sup _{t>r}\|f\|_{L_{p}, w^{p}} w(B(u, t))^{-\frac{1}{q}} \\
& \lesssim \sup _{u \in \mathbb{H}_{n}, r>0} \varphi_{1}(u, r)^{-1}\|w\|_{L_{q}(B(u, r))}^{-1}\|f\|_{L_{p}, w^{p}} \\
& \lesssim\|f\|_{M_{p, \varphi_{1}}\left(w^{p}\right)},
\end{aligned}
$$

and for $p=1$

$$
\begin{aligned}
\left\|M_{\alpha} f\right\|_{W M_{q, \varphi_{2}}\left(w^{q}\right)} & \lesssim \sup _{u \in \mathbb{H}_{n}, r>0} \varphi_{2}(u, r)^{-1} \sup _{t>r}\|f\|_{L_{1}, w} w(B(u, t))^{-\frac{1}{q}} \\
& \lesssim \sup _{u \in \mathbb{H}_{n}, r>0} \varphi_{1}(u, r)^{-1}\|w\|_{L_{q}(B(u, r))}^{-1}\|f\|_{L_{1}, w} \\
& \lesssim\|f\|_{M_{p, \varphi_{1}}\left(w^{p}\right)} .
\end{aligned}
$$

Remark 3.1 Note that, in the case $w \equiv 1$, Theorems 3.1 and 3.2 were proved in [14], see also $[2,8-10]$.

\section{Higher order fractional maximal commutator operators in the spaces $M_{p, \varphi}\left(\mathbb{H}_{n}, w\right)$}

In this section, we shall give the Spanne-Guliyev type boundedness of the higher order fractional maximal commutator operator $M_{b, \alpha, k}$ on the generalized weighted Morrey spaces $M_{p, \varphi}\left(\mathbb{H}_{n}, w\right)$. In the case of $\mathbb{R}^{n}$ Spanne-Guliyev type result for the operator $M_{\alpha}$ in the space $M_{p, \varphi}\left(\mathbb{R}^{n}, w\right)$ was proved in [22], see also [1,18,21].

We recall the definition of the space of $B M O\left(\mathbb{H}_{n}\right)$.

Definition 4.1 Suppose that $b \in L_{1}^{\mathrm{loc}}\left(\mathbb{H}_{n}\right)$, and let

$$
\|b\|_{*}=\sup _{u \in \mathbb{H}_{n}, r>0} \frac{1}{|B(u, r)|} \int_{B(u, r)}\left|b(v)-b_{B(u, r)}\right| d V(v)<\infty
$$

where

$$
b_{B(u, r)}=\frac{1}{|B(u, r)|} \int_{B(u, r)} b(v) d V(v) .
$$

Define

$$
B M O\left(\mathbb{H}_{n}\right)=\left\{b \in L_{1}^{\text {loc }}\left(\mathbb{H}_{n}\right):\|b\|_{*}<\infty\right\} .
$$

Modulo constants, the space $B M O\left(\mathbb{H}_{n}\right)$ is a Banach space with respect to the norm $\|\cdot\|_{*}$.

Lemma 4.1 [25] Let $w \in A_{\infty}$. Then the norm $\|\cdot\|_{*}$ is equivalent to the norm

$$
\|b\|_{*, w}=\sup _{u \in \mathbb{H}_{n}, r>0} \frac{1}{w(B(u, r))} \int_{B(u, r)}\left|b(v)-b_{B(u, r), w}\right| w(y) d V(v),
$$

where

$$
b_{B(u, r), w}=\frac{1}{w(B(u, r))} \int_{B(u, r)} b(v) w(v) d V(v) .
$$

The following lemma is proved in [15]. 


\section{Lemma 4.2}

1 Let $w \in A_{\infty}$ and $b \in B M O\left(\mathbb{H}_{n}\right)$. Let also $1 \leq p<\infty, u \in \mathbb{H}_{n}, k>0$ and $r_{1}, r_{2}>0$. Then,

$$
\left(\frac{1}{w\left(B\left(u, r_{1}\right)\right)} \int_{B\left(u, r_{1}\right)}\left|b(v)-b_{B\left(u, r_{2}\right), w}\right|^{k p} w(v) d V(v)\right)^{\frac{1}{p}} \leq C\left(1+\left|\ln \frac{r_{1}}{r_{2}}\right|\right)^{k}\|b\|_{*}^{k},
$$

where $C>0$ is independent of $f, w, u, r_{1}$ and $r_{2}$.

2 Let $w \in A_{p}$ and $b \in B M O\left(\mathbb{H}_{n}\right)$. Let also $1<p<\infty, u \in \mathbb{H}_{n}, k>0$ and $r_{1}, r_{2}>0$.

Then,

$$
\begin{aligned}
\left(\frac{1}{w^{-p^{\prime}}\left(B\left(u, r_{1}\right)\right)} \int_{B\left(u, r_{1}\right)}\left|b(v)-b_{B\left(u, r_{2}\right), w}\right|^{k p^{\prime}}\right. & \left.w(v)^{-p^{\prime}} d V(v)\right)^{\frac{1}{p^{\prime}}} \\
& \leq C\left(1+\left|\ln \frac{r_{1}}{r_{2}}\right|\right)^{k}\|b\|_{*}^{k},
\end{aligned}
$$

where $C>0$ is independent of $b, w, u, r_{1}$ and $r_{2}$.

The following lemma is valid.

Remark 4.1 $[6,31](1)$ Let $b \in B M O\left(\mathbb{H}_{n}\right)$. Then

$$
\|b\|_{*} \approx \sup _{u \in \mathbb{H}_{n}, r>0}\left(\frac{1}{|B(u, r)|} \int_{B(u, r)}\left|b(v)-b_{B(u, r)}\right|^{p} d V(v)\right)^{\frac{1}{p}}
$$

for $1<p<\infty$.

(2) Let $b \in B M O\left(\mathbb{H}_{n}\right)$. Then there is a constant $C>0$ such that

$$
\left|b_{B(u, r)}-b_{B(u, \tau)}\right| \leq C\|b\|_{*} \log \frac{\tau}{r} \text { for } 0<2 r<\tau,
$$

where $C$ is independent of $f, u, r$ and $\tau$.

The commutator generated by $b \in L_{1}^{\text {loc }}\left(\mathbb{R}^{n}\right)$ and the operator $M_{\alpha}$ is defined by

$$
M_{b, \alpha}(f)(u)=\sup _{r>0}|B(u, r)|^{-1+\frac{\alpha}{Q}} \int_{B(u, r)}|b(u)-b(v)||f(v)| d V(v) .
$$

For a positive integer $k$ and a function $b$, the $k$ th-order fractional maximal commutator $M_{b, \alpha, k}$ (see [14]) is defined by

$$
M_{b, \alpha, k}(f)(u)=\sup _{r>0}|B(u, r)|^{-1+\frac{\alpha}{Q}} \int_{B(u, r)}|b(u)-b(v)|^{k}|f(v)| d V(v) .
$$

The following Guliyev weighted local estimates are valid (see [15]).

Theorem 4.1 Let $1<p<q<\infty, 0<\alpha<\frac{Q}{p}$, $\frac{1}{q}=\frac{1}{p}-\frac{\alpha}{Q}, b \in B M O\left(\mathbb{H}_{n}\right)$, and $w \in A_{p, q}\left(\mathbb{H}_{n}\right)$. Then the inequality

$$
\begin{aligned}
& \left\|M_{b, \alpha, k} f\right\|_{L_{q, w}(B(u, r))} \\
& \lesssim\|b\|_{*}^{k} w^{q}(B(u, r))^{\frac{1}{q}} \sup _{t>2 r} \ln ^{k}\left(e+\frac{t}{r}\right) w^{q}(B(u, t))^{-\frac{1}{q}}\|f\|_{L_{p, w}(B(u, t))}
\end{aligned}
$$

holds for any ball $B(u, r)$ and for all $f \in L_{p, w^{p}}^{l o c}\left(\mathbb{H}_{n}\right)$. 
Proof. Let $1<p<q<\infty, 0<\alpha<\frac{Q}{p}, \frac{1}{q}=\frac{1}{p}-\frac{\alpha}{Q}$. We write $f$ as

$$
f=f_{1}+f_{2}, \quad f_{1}(v)=f(v) \chi_{2 B}(v), \quad f_{2}(v)=f(v) \chi_{\mathrm{c}_{(2 B)}}(v) .
$$

Hence,

$$
\left\|M_{b, \alpha, k} f\right\|_{L_{q, w}(B)} \leq\left\|M_{b, \alpha, k} f_{1}\right\|_{L_{q, w}(B)}+\left\|M_{b, \alpha, k} f_{2}\right\|_{L_{q, w}(B)} .
$$

From the boundedness of $M_{b, \alpha, k}$ from $L_{p, w^{p}}\left(\mathbb{H}_{n}\right)$ to $L_{q, w^{q}}\left(\mathbb{H}_{n}\right)$ (see [3,29]) it follows that

$$
\left\|M_{b, \alpha, k} f_{1}\right\|_{L_{q, w^{q}}(B)} \leq\left\|M_{b, \alpha, k} f_{1}\right\|_{L_{q, w^{q}}} \lesssim\|b\|_{*}^{k}\left\|f_{1}\right\|_{L_{p, w^{p}}}=\|b\|_{*}^{k}\|f\|_{L_{p, w^{p}}(2 B)} .
$$

Let $\zeta=(h, \tau)$ be an arbitrary point in $B \equiv B(u, r)$. If $B(\zeta, t) \cap{ }^{\mathrm{c}} B(u, 2 r) \neq \varnothing$, then $t>r$. Indeed, if $v \in B(\zeta, t) \cap{ }^{C} B(u, 2 r)$, then we get $t>\left|\zeta^{-1} v\right| \geq\left|v^{-1} u\right|-\left|\zeta^{-1} u\right|>$ $2 r-r=r$.

On the other hand, $B(\zeta, t) \cap{ }^{\complement} B(u, 2 r) \subset B(u, 2 t)$. Indeed, if $v \in B(\zeta, t) \cap{ }^{\complement} B(u, 2 r)$, then we get $\left|v^{-1} u\right| \leq\left|\zeta^{-1} v\right|+\left|\zeta^{-1} u\right|<t+r<2 t$. Hence, for all $\zeta \in B$

$$
\begin{aligned}
M_{b, \alpha, k} f_{2}(\zeta) & =\sup _{t>0}|B(\zeta, t)|^{-1+\frac{\alpha}{Q}} \int_{B(\zeta, t)}|b(v)-b(\zeta)|^{k}\left|f_{2}(v)\right| d V(v) \\
& =\sup _{t>0}|B(\zeta, t)|^{-1+\frac{\alpha}{Q}} \int_{B(\zeta, t) \cap^{\complement} B(u, 2 r)}|b(v)-b(\zeta)|^{k}|f(v)| d V(v) \\
& \lesssim \sup _{t>r}|B(u, 2 t)|^{-1+\frac{\alpha}{Q}} \int_{B(u, 2 t)}|b(v)-b(\zeta)|^{k}|f(v)| d V(v) \\
& =\sup _{t>2 r}|B(u, 2 t)|^{-1+\frac{\alpha}{Q}} \int_{B(u, t)}|b(v)-b(\zeta)|^{k}|f(v)| d V(v) .
\end{aligned}
$$

Therefore, for all $\zeta \in B$ we have

$$
M_{b, \alpha, k} f_{2}(\zeta) \lesssim \sup _{t>2 r}|B(u, 2 t)|^{-1+\frac{\alpha}{Q}} \int_{B(u, t)}|b(v)-b(\zeta)|^{k}|f(v)| d V(v) .
$$

Thus, the function $M_{\alpha} f_{2}(\zeta)$, with fixed $u$ and $r$, is dominated by the expression not depending on $\zeta$. Then

$$
\begin{aligned}
& \left\|M_{b, \alpha, k} f_{2}\right\|_{L_{q, w}(B)} \\
& \lesssim\left(\int_{B}\left(\sup _{t>2 r}|B(u, t)|^{-1+\frac{\alpha}{Q}} \int_{B(u, t)}|b(v)-b(\zeta)|^{k}|f(v)| d V(v)\right)^{q} w^{q}(\zeta) d V(\zeta)\right)^{\frac{1}{q}} \\
& \lesssim\left(\int_{B}\left(\sup _{t>2 r}|B(u, t)|^{-1+\frac{\alpha}{Q}} \int_{B(u, t)}\left|b(v)-b_{B(u, r), w}\right|^{k}|f(v)| d V(v)\right)^{q} w^{q}(\zeta) d V(\zeta)\right)^{\frac{1}{q}} \\
& +\left(\int_{B}\left(\sup _{t>2 r}|B(u, t)|^{-1+\frac{\alpha}{Q}} \int_{B(u, t)}\left|b(\zeta)-b_{B(u, r), w}\right|^{k}|f(v)| d V(v)\right)^{q} w^{q}(\zeta) d V(\zeta)\right)^{\frac{1}{q}} \\
& =J_{1}+J_{2} .
\end{aligned}
$$


Let us estimate $J_{1}$. Applying Hölder's inequality and by Lemma 4.2 we get

$$
\begin{array}{r}
J_{1}=w^{q}(B(u, r))^{\frac{1}{q}} \sup _{t>2 r}|B(u, t)|^{-1+\frac{\alpha}{Q}} \int_{B(u, t)}\left|b(v)-b_{B(u, r), w}\right|^{k}|f(v)| d V(v) \\
\approx w^{q}(B(u, r))^{\frac{1}{q}} \sup _{t>2 r} t^{\alpha-Q} \int_{B(u, t)}\left|b(v)-b_{B(u, r), w}\right|^{k}|f(v)| d V(v) \\
\leq\left(w^{q}(B(u, r))\right)^{\frac{1}{q}} \sup _{t>2 r} t^{\alpha-Q}\left(\int_{B(u, t)}\left|b(v)-b_{B(u, r), w}\right|^{k p^{\prime}} w(v)^{-p^{\prime}} d V(v)\right)^{\frac{1}{p^{\prime}}}\|f\|_{L_{p, w} p(B(u, t))} \\
\lesssim\|b\|_{*}^{k} w^{q}(B(u, r))^{\frac{1}{q}} \sup _{t>2 r} t^{\alpha-Q}\left(1+\ln \frac{t}{r}\right)^{k}\left\|w^{-1}\right\|_{L_{p^{\prime}}(B(u, t))}\|f\|_{L_{p, w} p(B(u, t))} \\
\lesssim\|b\|_{*}^{k} w^{q}(B(u, r))^{\frac{1}{q}} \sup _{t>2 r} t^{\alpha-Q}\left(1+\ln \frac{t}{r}\right)^{k}\left(w^{q}(B(u, t))\right)^{-\frac{1}{q}} t^{\frac{Q}{q}+\frac{Q}{p^{\prime}}}\|f\|_{L_{p, w}(B(u, t))} \\
=\|b\|_{*}^{k} w^{q}(B(u, r))^{\frac{1}{q}} \sup _{t>2 r} \ln ^{k}\left(e+\frac{t}{r}\right) w^{q}(B(u, t))^{-\frac{1}{q}}\|f\|_{L_{p, w}(B(u, t))} .
\end{array}
$$

In order to estimate $I_{2}$ we get

$$
\begin{array}{r}
J_{2}=\left(\int_{B}\left(\sup _{t>2 r}|B(u, t)|^{-1+\frac{\alpha}{Q}} \int_{B(u, t)}\left|b(\zeta)-b_{B(u, r), w}\right|^{k}|f(v)| d V(v)\right)^{q} w^{q}(\zeta) d V(\zeta)\right)^{\frac{1}{q}} \\
\approx\left(\int_{B}\left|b(\zeta)-b_{B(u, r), w}\right|^{k q} w^{q}(\zeta) d V(\zeta)\right)^{\frac{1}{q}} \sup _{t>2 r} t^{\alpha-Q} \int_{B(u, t)}|f(v)| d V(v) .
\end{array}
$$

According to the first part of Lemma 4.2, we get

$$
\begin{array}{r}
J_{2} \lesssim\|b\|_{*}^{k}\left(1+\ln \frac{r}{r}\right)^{k} w^{q}(B(u, r))^{\frac{1}{q}} \sup _{t>2 r} t^{\alpha-Q} \int_{B(u, t)}|f(v)| d V(v) \\
\leq\|b\|_{*}^{k} w^{q}(B(u, r))^{\frac{1}{q}} \sup _{t>2 r} t^{\alpha-Q}\|f\|_{L_{p, w^{p}}(B(u, t))}\left\|w^{-1}\right\|_{L_{p^{\prime}}(B(u, t))} \\
=\|b\|_{*}^{k} w^{q}(B(u, r))^{\frac{1}{q}} \sup _{t>2 r} \ln ^{k}\left(e+\frac{t}{r}\right) w^{q}(B(u, t))^{-\frac{1}{q}}\|f\|_{L_{p, w} p(B(u, t))} .
\end{array}
$$

Summing up $J_{1}$ and $J_{2}$, for all $p \in(1, \infty)$ we get

$$
\begin{aligned}
& \left\|M_{b, \alpha, k} f_{2}\right\|_{L_{q, w}(B)} \lesssim \\
& \|b\|_{*}^{k} w^{q}(B(u, r))^{\frac{1}{q}} \sup _{t>2 r} \ln ^{k}\left(e+\frac{t}{r}\right) w^{q}(B(u, t))^{-\frac{1}{q}}\|f\|_{L_{p, w^{p}}(B(u, t))} .
\end{aligned}
$$

Finally, from (4.4) and (4.5) we get

$$
\begin{aligned}
\left\|M_{b, \alpha, k} f\right\|_{L_{q, w^{q}}(B)} \lesssim\|b\|_{*}^{k}\left(w^{q}(B(u, r))\right)^{\frac{1}{q}} \sup _{t \geq r}\|f\|_{L_{p, w}(B(u, t))}\left(w^{q}(B(u, t))\right)^{-\frac{1}{q}} \\
+\|b\|_{*}^{k} w^{q}(B(u, r))^{\frac{1}{q}} \sup _{t>2 r} \ln ^{k}\left(e+\frac{t}{r}\right) w^{q}(B(u, t))^{-\frac{1}{q}}\|f\|_{L_{p, w}(B(u, t))} \\
\lesssim\|b\|_{*}^{k} w^{q}(B(u, r))^{\frac{1}{q}} \sup _{t>2 r} \ln ^{k}\left(e+\frac{t}{r}\right) w^{q}(B(u, t))^{-\frac{1}{q}}\|f\|_{L_{p, w}(B(u, t))} .
\end{aligned}
$$

For the operator $M_{b, \alpha, k}$ the following Spanne-Guliyev type result on the space $M_{p, \varphi}(w)$ is valid. 
Theorem 4.2 Let $1<p<q<\infty, 0<\alpha<\frac{Q}{p}, \frac{1}{q}=\frac{1}{p}-\frac{\alpha}{Q}, w \in A_{p, q}\left(\mathbb{H}_{n}\right), b \in$ $B M O\left(\mathbb{H}_{n}\right)$ and $\left(\varphi_{1}, \varphi_{2}\right)$ satisfy the condition

$$
\sup _{t>r} \ln ^{k}\left(e+\frac{t}{r}\right) \frac{\underset{t<s<\infty}{\operatorname{ess} \inf } \varphi_{1}(u, s)\left(w^{p} B((u, s))\right)^{1 / p}}{\left(w^{q}(B(u, t))\right)^{1 / q}} \leq C \varphi_{2}(u, r),
$$

where $C$ does not depend on $u$ and $r$. Then the operator $M_{b, \alpha, k}$ is bounded from $M_{p, \varphi_{1}}\left(w^{p}\right)$ to $M_{q, \varphi_{2}}\left(w^{q}\right)$. Moreover,

$$
\left\|M_{b, \alpha, k} f\right\|_{M_{q, \varphi_{2}}\left(w^{q}\right)} \lesssim\|b\|_{*}^{k}\|f\|_{M_{p, \varphi_{1}}\left(w^{p}\right)} .
$$

Proof. Using the Theorem 2.1 and the Theorem 4.1 we have

$$
\begin{aligned}
& \left\|M_{b, \alpha, k} f\right\|_{M_{q, \varphi_{2}}\left(w^{q}\right)}=\sup _{u \in \mathbb{H}_{n}, r>0} \varphi_{2}(u, r)^{-1} w^{q}(B(u, r))^{\frac{1}{q}}\left\|M_{b, \alpha, k} f\right\|_{L_{q, w}{ }^{q} B(u, r)} \\
& \lesssim\|b\|_{*}^{k} \sup _{u \in \mathbb{H}_{n}, r>0} \varphi_{2}(u, r)^{-1} \sup _{t>2 r} \ln ^{k}\left(e+\frac{t}{r}\right) w^{q}(B(u, t))^{-\frac{1}{q}}\|f\|_{L_{p, w}(B(u, t))} \\
& \lesssim\|b\|_{*}^{k} \sup _{u \in \mathbb{H}_{n}, r>0} \varphi_{1}(u, r)^{-1}\left(w^{p}(B(u, r))\right)^{-\frac{1}{p}}\|f\|_{L_{p, w}(B(u, r))} \\
& =\|b\|_{*}^{k}\|f\|_{M_{p, \varphi_{1}}\left(w^{p}\right)} .
\end{aligned}
$$

Remark 4.2 Note that, in the case $w \equiv 1$, Theorems 4.1 and 4.2 were proved in [14], see also [2,8-10].

Acknowledgements. The author thanks the referees for careful reading of the paper and useful comments. The author would like to express his deep gratitude to corresponding member of the National Academy of Sciences of Azerbaijan, Professor Vagif S. Guliyev for his attention to this work.

\section{References}

1. Akbulut, A., Hamzayev, V.H., Safarov, Z.V.: Rough fractional multilinear integral operators on generalized weighted Morrey spaces, Azerb. J. Math. 6 (2), 128-142 (2016).

2. Azizov, C.V.: Fractional integral operator and its higher order commutators in generalized weighted Morrey spaces on Heisenberg group, Trans. Natl. Acad. Sci. Azerb. Ser. Phys.-Tech. Math. Sci. 39 (4), Mathematics, 25-36 (2019).

3. Bernardis, A., Hartzstein, S., Pradolini, G.: Weighted inequalities for commutators of fractional integrals on spaces of homogeneous type, J. Math. Anal. Appl. 322, 825-846 (2006).

4. Burenkov, V., Gogatishvili, A., Guliyev, V.S., Mustafayev, R.: Boundedness of the fractional maximal operator in local Morrey-type spaces, Complex Var. Elliptic Equ. 55 (810), 739-758 (2010).

5. Capogna, L., Danielli, D., Pauls, S., Tyson, J.: An introduction to the Heisenberg group and the sub-Riemannian isoperimetric problem, Progr. Math. 259, Birkhauser, Basel, (2007).

6. Folland, G.B., Stein, E.M.: Hardy spaces on homogeneous groups. Mathematical Notes, 28, Princeton Univ. Press, Princeton, (1982).

7. Folland, G.B., Stein, E.M.: Estimates for the $\partial_{b}$-complex and analysis on the Heisenberg group, Comm Pure Appl Math, 27, 429-522 (1974). 
8. Eroglu, A., Azizov, C.V.: A note of the fractional integral operators in generalized Morrey spaces on the Heisenberg group, Trans. Natl. Acad. Sci. Azerb. Ser. Phys.-Tech. Math. Sci. 37 (1), Mathematics, 86-91 (2017).

9. Eroglu, A., Azizov, C.V., Guliyev, V.S.: Fractional maximal operator and its commutators in generalized Morrey spaces on Heisenberg group, Proc. Inst. Math. Mech. Natl. Acad. Sci. Azerb. 44 (2), 304-317 (2018).

10. Eroglu, A., Isayev, F.A., Namazov, F.M.: Fractional integral associated to Schrodinger operator on the Heisenberg groups in vanishing generalized Morrey spaces, Trans. Natl. Acad. Sci. Azerb. Ser. Phys.-Tech. Math. Sci. 38 (4), Mathematics, 54-62 (2018).

11. Guliyev, V.S.: Integral operators on function spaces on the homogeneous groups and on domains in $\mathbb{G}$, Doctor's degree dissertation, Moscow, Mat. Inst. Steklov, 1994, 1-329. (Russian)

12. Guliyev, V.S.: Function spaces, integral operators and two weighted inequalities on homogeneous groups. Some applications, Baku. 1999, 1-332. (Russian)

13. Guliyev, V.S.: Boundedness of the maximal, potential and singular operators in the generalized Morrey spaces, J. Inequal. Appl. 2009, Art. ID 503948, 20 pp.

14. Guliyev, V.S., Akbulut, A., Mammadov, Y.Y.: Boundedness of fractional maximal operator and their higher order commutators in generalized Morrey spaces on Carnot groups, Acta Math. Sci. Ser. B Engl. Ed. 33 (5), 1329-1346 (2013).

15. Guliyev, V.S.: Generalized weighted Morrey spaces and higher order commutators of sublinear operators, Eurasian Math. J. 3 (3), 33-61 (2012).

16. Guliyev, V.S., Karaman, T., Mustafayev, R.Ch., Serbetci, A.: Commutators of sublinear operators generated by Calderón-Zygmund operator on generalized weighted Morrey spaces, Czechoslovak Math. J. 64 (139) (2), 1-22 (2014).

17. Guliyev, V.S., Alizadeh, F.Ch.: Multilinear commutators of Calderon-Zygmund operator on generalized weighted Morrey spaces, J. Funct. Spaces, 2014, Art. ID 710542, 9 pp.

18. Guliyev, V.S., Omarova, M.N.: Multilinear singular and fractional integral operators on generalized weighted Morrey spaces, Azerb. J. Math. 5 (1), 104-132 (2015).

19. Guliyev, V.S., Hamzayev, V.H.: Rough singular integral operators and its commutators on generalized weighted Morrey spaces, Math. Ineq. Appl. 19 (3), 863-881 (2016).

20. Guliyev, V.S., Omarova, M.N., Softova, L.: The Dirichlet problem in a class of generalized weighted Morrey spaces, Proc. Inst. Math. Mech. Natl. Acad. Sci. Azerb. 45 (2), 270-285 (2019).

21. Hamzayev, V.H.: Sublinear operators with rough kernel generated by CalderonZygmund operators and their commutators on generalized weighted Morrey spaces, Trans. Natl. Acad. Sci. Azerb. Ser. Phys.-Tech. Math. Sci. 38 (1), Mathematics, 79-94 (2018).

22. Ismayilova, A.F.: Fractional maximal operator and its commutators on generalized weighted Morrey spaces, Trans. Natl. Acad. Sci. Azerb. Ser. Phys.-Tech. Math. Sci. 39 (4), Mathematics, 84-95 (2019).

23. Komori, Y., Shirai, S.: Weighted Morrey spaces and a singular integral operator, Math. Nachr. 282 (2), 219-231 (2009).

24. Muckenhoupt, B.: Weighted norm inequalities for the Hardy maximal function, Trans. Amer. Math. Soc. 165, 207-226 (1972).

25. Muckenhoupt, B., Wheeden, R.: Weighted norm inequalities for fractional integrals, Trans. Amer. Math. Soc. 192, 261-274 (1974).

26. Mizuhara, T.: Boundedness of some classical operators on generalized Morrey spaces, Harmonic Analysis (S. Igari, Editor), ICM 90 Satellite Proceedings, Springer - Verlag, Tokyo, 183-189 (1991).

27. Morrey, C.B.: On the solutions of quasi-linear elliptic partial differential equations, Trans. Amer. Math. Soc. 43, 126-166 (1938). 
28. Nakai, E.: Hardy-Littlewood maximal operator, singular integral operators and Riesz potentials on generalized Morrey spaces, Math. Nachr. 166, 95-103 (1994).

29. Perez, C., Wheeden, R.: Uncertainty principle estimates for vector fields, J. Funct. Anal. 181, 146-188 (2001).

30. Sawano, Y.: A thought on generalized Morrey spaces, J. Indonesian Math. Soc. 25 (3), 210-281 (2019).

31. Stein, E.M.: Harmonic Analysis: Real-Variable Methods, Orthogonality and Oscillatory Integrals, Princeton Univ. Press, Princeton, NJ, (1993). 